\title{
Oxidative stress markers, cognitive functions, and psychosocial functioning in bipolar disorder: an empirical cross-sectional study
}

\author{
Ömer Aydemir, Zeynep Çubukçuoğlu, Soner Erdin, Cumhur Taş, Ece Onur, Michael Berk \\ Department of Psychiatry, Celal Bayar University, Manisa, Turkey.
}

\begin{abstract}
Objective: This study aimed to evaluate the relationship between oxidative stress markers and cognitive functions and domains of psychosocial functioning in bipolar disorder.

Methods: Oxidative stress markers, cognitive functions, and domains of psychosocial functioning were evaluated in 51 patients with bipolar disorder who were in remission. Correlation analyses between these parameters were calculated with data controlled for duration of illness and number of episodes.

Results: There was no statistically significant correlation between oxidative stress markers and cognitive functions. In terms of psychosocial functioning, significant correlations were found between malondialdehyde and sense of stigmatization $(r=-0.502)$; household activities and superoxide dismutase $(r=0.501)$; participation in social activities and nitric oxide $(r=0.414)$; hobbies and leisure time activities and total glutathione $(r=-0.567)$, superoxide dismutase $(r=0.667)$, and neurotrophin 4 $(r=0.450)$; and taking initiative and self-sufficiency and superoxide dismutase $(r=0.597)$. There was no correlation between other domains of psychosocial functioning and oxidative stress markers.

Conclusion: These results imply that oxidative stress markers do not appear to correlate clearly with cognitive impairment and reduced psychosocial functioning. However, there were some associations between selected oxidative markers and activity-oriented functional markers. This may represent a true negative association, or may be an artifact of oxidative stress being a state rather than a trait marker.
\end{abstract}

Keywords: Bipolar disorder; biomarkers; neurocognition; psychosocial functioning; oxidative stress

\section{Introduction}

There is a theoretical link between biomarkers associated with neuroprogression, including inflammation, oxidative stress, mitochondrial dysfunction, apoptosis, and decreased neurogenesis, and the decline in functioning, cognition, and treatment response that is seen in bipolar disorder. This process has been seen as part of the process of staging, and is linked to the notion of allostatic load. ${ }^{1-3}$ The theoretical background of the model is based on the progressively deteriorating course of bipolar disorder with recurrent episodes. Regarding allostatic load, Kapczinski et al. ${ }^{4}$ highlighted the role of oxidative stress, immune dysfunction, hormones, and dysregulation of neurotrophins and the cumulative effect of medical comorbidity, longer duration of illness, and multiple mood episodes. Grande et al. ${ }^{3}$ suggested that disease progression is associated with a change in biological markers; that is, as allostasis builds and accumulates, bipolar disorder is progressively associated with worse outcomes. Furthermore, Vieta et al. ${ }^{5}$ noted that cognitive impairment,

Correspondence: Omer Aydemir, Celal Bayar Uni., School of Medicine, 45030, Manisa, Turkey.

E-mail: soaydemir@yahoo.com

Submitted Nov 01 2013, accepted Jan 082014. especially verbal memory function, predicts reduced psychosocial functioning, and neurotrophins and inflammatory cytokines such as tumor necrosis factor-alpha (TNF-alpha) can be considered as neurobiological markers implicated in neurodegeneration and cognitive impairment in bipolar disorder. This model also constitutes the basis of staging in bipolar disorder. ${ }^{4,6}$ However, these correlations are not demonstrated in empirical studies.

This study aimed to evaluate the relationship between oxidative stress markers and cognitive functions and domains of psychosocial functioning to test the contribution of oxidative stress to the allostatic load model in bipolar disorder, using an empirical study design. The primary hypothesis of the study was that correlations would be found between oxidative stress markers and impairment in cognitive functions. Potential correlations between oxidative stress markers and psychosocial functions were secondary outcomes.

\section{Methods}

\section{Participants}

The study was carried out among participants followed up at the Unit for Mood Disorders of Celal Bayar University 
School of Medicine, Manisa, Turkey, between February 2010 and November 2011. The inclusion criteria were age between 18 and 65 years, being in remission for at least 6 months, having a diagnosis of bipolar disorder according to DSM-IV criteria, and demonstrating sufficient physical and cognitive ability to comply with the study protocol. The exclusion criteria were having any Axis I psychiatric disorder other than bipolar disorder, alcohol or any other substance misuse, any neurological or organic diagnosis, and using any medication other than those prescribed for bipolar disorder, including antioxidants (e.g., vitamin $\mathrm{C}$ or $\mathrm{E}$, antioxidant treatment such as $\mathrm{N}$-acetylcysteine). All diagnoses were made by means of an SCID-CV interview. Asymptomatic mood state was confirmed by a 17-item Hamilton Depression Rating Scale (HAM-D) score less than 7 and a Young Mania Rating Scale (YMRS) score less than 4 at the time of the SCID-CV interview. All subjects had been asymptomatic for at least 6 months on the basis of clinician notes and SCID-CV interview findings. Additional clinical information (i.e., onset of illness, number of episodes and hospitalizations) was obtained both from clinical charts and directly in patient interviews. The patients were assessed cross-sectionally. As a result, 51 individuals with a diagnosis of bipolar disorder were included in the study. All of the participants were on psychotropic medication: $43.1 \%(n=22)$ were on monotherapy and the rest $(n=29)$ were receiving combination therapy. Only five of the patients were not receiving any mood stabilizer, while $11(21.5 \%)$ were receiving second-generation antipsychotics. In addition, 50 healthy control subjects without any psychiatric, neurological, or organic disorders were included in the study. The study was approved by the local ethics committee of Celal Bayar University (14.05.20090032). All subjects gave written informed consent for participation.

\section{Measures}

\section{Mood symptoms}

The 17-item HAM-D, in its Turkish version as validated by Aydemir et al., ${ }^{7}$ was used to assess depressive symptoms. The YMRS, in its Turkish version as validated by Karadag et al., ${ }^{8}$ was used to assess manic symptoms.

\section{Cognitive functions}

The cognitive test battery was selected according to the ISBD-BANC. ${ }^{9}$ The Continuous Performance Test was used to assess attention; the Wisconsin Card Sorting Test, Stroop Test, and Trail Making Test (part B) to assess executive functioning and working memory; the Trail Making Test (part A), to assess processing speed; the Rey Auditory Verbal Learning Test and Serial Digit Learning Test, to assess verbal learning and memory; and the Judgment of Line Orientation Test, to assess visuospatial function.

\section{Oxidative stress markers}

For the assessment of oxidative stress, we evaluated the levels of total glutathione (GSH), superoxide dismutase (SOD), nitric oxide (NO) and malondialdehyde (MDA), which are the major components of oxidant-antioxidant systems. Levels of neurotrophin 4 (NT-4), which has a suggested protective role against neuronal oxidative stress, and of homocysteine, a neurotoxic amino acid, were also assayed.

\section{Psychosocial functions}

The Bipolar Disorder Functioning Questionnaire (BDFQ) was administered to assess psychosocial functioning. The BDFQ was developed and validated by the Scientific Committee of Mood Disorders, Psychiatric Association of Turkey. ${ }^{10}$ It is a self-rated, three-point Likert type scale with 11 subscales, namely emotional functioning, intellectual functioning, sexual functioning, sense of stigmatization, social withdrawal, household activities, relations with friends, participation in social activities, daily activities and hobbies, taking initiative and self-sufficiency, and occupation. Higher scores indicate better psychosocial functioning.

\section{Procedure}

After written informed consent was obtained, blood samples were collected from the volunteers, after which all the mood scales and cognitive tests were performed. Serum SOD, NT-4, homocysteine, vitamin B12, and folic acid levels, plasma MDA levels, and whole blood GSH levels were measured with commercial kits in peripheral venous blood samples collected from all volunteers. ${ }^{11,12}$ SOD levels were analyzed by the ELISA method (Chemical Company, Michigan, U.S.) using the tetrazolium salt to detect superoxide radical produced by the xanthine oxidase enzyme. NT-4 levels were also analyzed by ELISA (Cusabio Biotech, Wuhan, China), using NT-4 specific antibodies on the wells. GSH and malondialdehyde levels were analyzed with the ImmuChrom GmbH systems in a Shimadzu Prominence high-performance liquid chromatography (HPLC) system. Vitamin B12 and folic acid levels were analyzed in an automated chemiluminescence system (Unicel DXI 800 Beckman Coulter), as were homocysteine levels (Immulite-2000, Diagnostic Products Corporation LA, U.S.). Serum NO levels were measured by the Griess method.

\section{Data analysis}

The allostatic load model of bipolar disorder is based on the concurrent total progressive deterioration in biomarkers, cognitive functions, and psychosocial functioning. Thus, these variables should correlate with each other independently of illness duration. In our statistical analyses, partial correlation coefficients were calculated to reveal the correlation between oxidative stress markers, cognitive functions, and psychosocial functioning 
domains, and the data were controlled for duration of illness and number of episodes, as there was variability among the sample. Since correlation coefficient values between 0.0 and 0.3 are considered weak, a correlation coefficient $(r) \geqslant 0.4$ was considered significant. The Student $t$-test was used for comparison of continuous variables between the bipolar disorder group and healthy control group. For this purpose, the significance level was set at $p<0.05$.

\section{Results}

The demographic and clinical features of the participants are given in Table 1. A comparison of oxidative stress markers between bipolar patients and healthy controls is shown in Table 2. MDA and NO levels were higher in the bipolar disorder group than in the control group, whereas SOD levels were lower in the bipolar disorder group than in the control group.

\section{Correlations between study variables}

There was no statistically significant correlation $(r \geqslant$ 0.40 ) between oxidative stress markers and cognitive functions (Table 3).

In terms of psychosocial functioning, there were six statistically significant correlations (Table 4) between oxidative stress markers and domains of psychosocial functioning. These significant correlations were between malondialdehyde and sense of stigmatization $(r=-0.502)$, household activities and superoxide dismutase $(r=$ $0.501)$, participation in social activities and nitric oxide $(r$ $=0.414)$, hobbies and leisure time activities and total glutathione $(r=-0.567)$, superoxide dismutase $(r=$ 0.667), and neurotrophin $4(r=0.450)$, and taking initiative and self-sufficiency and superoxide dismutase $(r=0.597)$. There was no correlation between other domains of psychosocial functioning and oxidative stress markers.

Table 1 Demographic and clinical features of the patients $(n=101)$

\begin{tabular}{lcc}
\hline & $\begin{array}{c}\text { Bipolar disorder } \\
\text { group }(\mathrm{n}=51)\end{array}$ & $\begin{array}{c}\text { Control } \\
\text { group }(\mathrm{n}=50)\end{array}$ \\
\hline Age (years) & $40.8 \pm 11.5$ & $39.8 \pm 11.2$ \\
Gender & & \\
$\quad$ Female & $24(47.1 \%)$ & $23(46.0 \%)$ \\
$\quad$ Male & $27(52.9 \%)$ & $27(54.0 \%)$ \\
Education (years) & $12.1 \pm 3.6$ & $12.0 \pm 3.7$ \\
Bipolar disorder type & & \\
$\quad$ Type 1 & $33(64.7 \%)$ & \\
$\quad$ Type 2 & $18(35.3 \%)$ & \\
Duration of illness (years) & $14.1 \pm 9.1$ & \\
Number of episodes & $5.1 \pm 4.0$ & \\
HAM-D & $0.9 \pm 1.8$ & \\
YMRS & $0.5 \pm 1.3$ &
\end{tabular}

HAM-D = Hamilton Depression Rating Scale; YMRS = Young Mania Rating Scale.
Table 2 Comparison of oxidative stress markers between the bipolar disorder and healthy control groups

\begin{tabular}{lcc}
\hline & $\begin{array}{c}\text { Bipolar disorder } \\
(\mathrm{n}=51)\end{array}$ & $\begin{array}{c}\text { Healthy controls } \\
(\mathrm{n}=50)\end{array}$ \\
\hline Glutathione $(\mathrm{mmol} / \mathrm{L})$ & $978.20 \pm 319.97$ & $1001.79 \pm 179.60$ \\
MDA $(\mathrm{mmol} / \mathrm{L})$ & $1.56 \pm 0.59^{*}$ & $1.04 \pm 0.28$ \\
NO $(\mathrm{mmol} / \mathrm{L})$ & $94.58 \pm 42.04^{*}$ & $64.07 \pm 38.36$ \\
SOD $(\mathrm{U} / \mathrm{ml})$ & $0.31 \pm 0.10^{*}$ & $0.39 \pm 0.15$ \\
NT-4 $(\mathrm{ng} / \mathrm{ml})$ & $6.30 \pm 4.92$ & $5.85 \pm 3.17$ \\
Homocysteine & $16.29 \pm 10.32$ & $17.81 \pm 13.12$ \\
(mmol/L) & & \\
Folic acid $(\mathrm{ng} / \mathrm{ml})$ & $5.83 \pm 2.69$ & $4.95 \pm 1.91$ \\
Vitamin B12 $(\mathrm{pg} / \mathrm{ml})$ & $234.16 \pm 150.64$ & $161.38 \pm 47.53$ \\
\hline
\end{tabular}

MDA = malondialdehyde; NO = nitric oxide; NT-4 = neurotrophin 4; $\mathrm{SOD}=$ superoxide dismutase.

$* p<0.001$.

\section{Discussion}

To our knowledge, this is the first empirical study to assess the correlation between oxidative stress markers and cognitive functions and domains of psychosocial functioning. In this study, we failed to demonstrate significant correlations between oxidative stress markers and cognitive functions or domains of psychosocial functioning. However, we were able to demonstrate some associations between selected oxidative markers and activity-oriented functional markers. Nevertheless, these correlations do not display a clear pattern.

Although it has been suggested in the literature that bipolar disorder has a favorable clinical course, it has been clearly demonstrated that many patients have residual subsyndromal symptoms between episodes. ${ }^{13}$ Furthermore, cognitive functions, especially verbal learning and memory, are impaired in some individuals with bipolar disorder, and duration of illness correlates negatively with cognitive impairment. ${ }^{14}$ Rosa et al. ${ }^{15}$ reported that patients with bipolar disorder showed worse performance in areas of psychosocial functioning, and one of the predictors was older age. Kapczinski et al. ${ }^{4}$ showed that biochemical markers may change significantly from the early to late stages of bipolar disorder. Thus, there is evidence that the course of bipolar disorder involves progressive impairment in multiple areas.

Even though longer duration of illness is an important predictor of worse outcome, there are other factors that seem to affect the outcome of bipolar disorder. Persistent subsyndromal depressive symptoms have substantial effects on cognitive ${ }^{14}$ and psychosocial functioning. ${ }^{16,17}$ Medication side effects may also worsen cognitive functions $^{15}$ and have some effects on psychosocial functioning. ${ }^{18}$ Previous psychotic symptoms seem to affect cognitive functions ${ }^{19}$ and quality of life. ${ }^{20}$ Thus, $^{2}$ many other factors impact outcome and staging in the clinical course of bipolar disorder.

In recent years, both Berk et al. ${ }^{21,22}$ and Kapczinski et al. ${ }^{6}$ have put forward models of staging in bipolar disorder. These models are all based on the course of the illness, both taking into account the clinical symptomatology and neuroprogression with biomarkers and cognitive impairment, in addition to psychosocial 
Table 3 Correlation coefficients between oxidative stress markers and cognitive tests in patients with bipolar disorder ( $\mathrm{n}=51$ )

\begin{tabular}{|c|c|c|c|c|c|c|c|}
\hline & Folic acid & Homocysteine & Glutathione & MDA & NO & SOD & NT-4 \\
\hline Stroop Test (time) & 0.184 & -0.084 & -0.012 & 0.085 & 0.125 & 0.031 & -0.055 \\
\hline Stroop Test (omissions) & -0.075 & -0.041 & -0.045 & -0.032 & -0.093 & -0.003 & 0.119 \\
\hline Serial Digit Learning Test & 0.153 & -0.065 & -0.107 & 0.004 & -0.010 & -0.060 & -0.096 \\
\hline Judgment of Line Orientation Test & 0.068 & 0.028 & 0.065 & 0.180 & -0.141 & 0.066 & -0.163 \\
\hline Wisconsin Card Sorting Test (hits) & 0.187 & -0.103 & 0.011 & -0.013 & -0.050 & 0.245 & -0.135 \\
\hline $\begin{array}{l}\text { Wisconsin Card Sorting Test } \\
\text { (perseverative errors) }\end{array}$ & -0.135 & 0.250 & 0.098 & 0.059 & 0.142 & -0.303 & -0.030 \\
\hline $\begin{array}{l}\text { Wisconsin Card Sorting Test } \\
\text { (categories) }\end{array}$ & 0.151 & -0.076 & 0.065 & 0.157 & -0.026 & 0.165 & -0.154 \\
\hline $\begin{array}{l}\text { Continuous Performance Test } \\
\text { (correct detection) }\end{array}$ & 0.217 & 0.161 & 0.084 & -0.219 & 0.012 & 0.011 & 0.064 \\
\hline $\begin{array}{l}\text { Continuous Performance Test } \\
\text { (omission errors) }\end{array}$ & 0.229 & 0.033 & -0.047 & -0.088 & 0.065 & -0.017 & 0.063 \\
\hline Trail Making Test part A (time) & -0.169 & 0.021 & -0.096 & -0.256 & 0.007 & -0.129 & 0.076 \\
\hline Trail Making Test part A (omissions) & -0.071 & -0.081 & -0.251 & -0.050 & -0.220 & 0.008 & 0.143 \\
\hline Trail Making Test part B (time) & -0.060 & 0.119 & -0.143 & -0.252 & -0.044 & 0.078 & 0.046 \\
\hline Trail Making Test part B (omissions) & -0.077 & 0.067 & -0.204 & -0.128 & 0.027 & -0.009 & 0.034 \\
\hline $\begin{array}{l}\text { Rey Auditory Verbal Learning Test } \\
\text { (immediate recall) }\end{array}$ & 0.077 & 0.022 & 0.247 & -0.043 & 0.005 & 0.090 & 0.081 \\
\hline $\begin{array}{l}\text { Rey Auditory Verbal Learning Test } \\
\text { (short delay recall) }\end{array}$ & 0.339 & -0.276 & 0.344 & -0.098 & 0.009 & 0.142 & -0.102 \\
\hline $\begin{array}{l}\text { Rey Auditory Verbal Learning Test } \\
\text { (long delay recall) }\end{array}$ & 0.334 & -0.235 & 0.339 & -0.092 & -0.023 & 0.075 & -0.110 \\
\hline $\begin{array}{l}\text { Rey Auditory Verbal Learning Test } \\
\text { (correct hits) }\end{array}$ & 0.305 & -0.210 & 0.348 & -0.036 & 0.033 & 0.001 & -0.130 \\
\hline $\begin{array}{l}\text { Rey Auditory Verbal Learning Test } \\
\text { (false positive errors) }\end{array}$ & -0.135 & 0.183 & -0.196 & -0.121 & 0.181 & -0.052 & 0.221 \\
\hline
\end{tabular}

$\mathrm{MDA}=$ malondialdehyde; $\mathrm{NO}=$ nitric oxide; NT-4 = neurotrophin $4 ; \mathrm{SOD}=$ superoxide dismutase .

functioning. Andreazza et al. $^{23}$ reported that peripheral oxidative imbalance takes place during the active phases of the illness, but is not clearly evident in euthymic individuals. These findings suggest that oxidative stress markers are more state markers than trait markers.

The results of the present study suggest that there is no correlation between oxidative stress markers and cognitive functions and domains of psychosocial functioning, after controlling for duration of illness and number of episodes. These results imply that oxidative stress is not associated with cognitive impairment and reduced psychosocial functioning. Even though there was a significant difference between the bipolar disorder group and the healthy controls in favor of increased oxidative stress in the former, this does not point to a progressive course, as the present study was cross-sectional. An alternate explanation is that oxidative stress is a state marker, associated with acute illness, and is not a reliable trait marker of the illness, and hence is quiescent in euthymia.

With regard to limitations, the sample size of the study limits the generalizability of the results. Since all patients were on medication, it is impossible to disregard the influence of pharmacotherapy on the results. The crosssectional design of the study is another limitation; further studies with longitudinal designs may help demonstrate and clarify the relationship between outcome criteria in bipolar disorder. The absence of neurocognitive and psychosocial function testing and the correlations

Table 4 Correlation coefficients between oxidative stress markers and psychosocial functioning domains in patients with bipolar disorder $(n=51)$

\begin{tabular}{|c|c|c|c|c|c|c|c|}
\hline & Folic acid & Homocysteine & Glutathione & MDA & $\mathrm{NO}$ & SOD & NT-4 \\
\hline Emotional functioning & -0.280 & -0.229 & 0.113 & -0.187 & 0.342 & 0.327 & 0.053 \\
\hline Intellectual functioning & -0.164 & 0.184 & -0.220 & -0.139 & -0.031 & 0.092 & -0.267 \\
\hline Sexual functioning & -0.012 & 0.272 & -0.224 & -0.130 & -0.038 & -0.045 & -0.274 \\
\hline Sense of stigmatization & -0.028 & 0.176 & 0.141 & $-0.502 *$ & -0.109 & -0.068 & -0.060 \\
\hline Social withdrawal & -0.305 & 0.035 & -0.246 & -0.210 & 0.000 & 0.324 & 0.223 \\
\hline Household activities & -0.134 & -0.047 & -0.239 & -0.266 & 0.057 & $0.501 *$ & 0.359 \\
\hline Relations with friends & -0.066 & -0.014 & -0.183 & 0.025 & 0.178 & 0.287 & 0.151 \\
\hline Participation in social activities & -0.031 & 0.094 & -0.211 & 0.228 & $0.414^{\dagger}$ & 0.421 & 0.245 \\
\hline Hobbies and leisure time activities & -0.156 & 0.175 & $-0.567^{*}$ & 0.154 & 0.208 & $0.667^{*}$ & $0.450^{\dagger}$ \\
\hline Taking initiative and self-sufficiency & -0.356 & 0.314 & -0.350 & 0.314 & -0.112 & $0.597^{*}$ & 0.162 \\
\hline Occupation & 0.137 & 0.201 & -0.190 & -0.207 & 0.187 & 0.118 & 0.089 \\
\hline
\end{tabular}

$\mathrm{MDA}=$ malondialdehyde; $\mathrm{NO}=$ nitric oxide; NT-4 = neurotrophin $4 ; \mathrm{SOD}=$ superoxide dismutase

$* p<0.001$

$\dagger \mathrm{p}<0.005$. 
between oxidative stress markers and these tests in the control group limit the interpretation of the results in the bipolar disorder group.

In conclusion, it has been previously suggested that oxidative stress is implicated in the process of neuroprogression of bipolar disorder, including structural brain changes, and consequent cognitive impairment and reduced psychosocial functioning. ${ }^{1}$ However, oxidative stress and measures of functioning did not correlate in the present study.

\section{Disclosure}

OA has participated in a multicenter study sponsored by AstraZeneca; has served as a speaker for Lundbeck, AstraZeneca, Janssen-Cilag, and Pfizer; and has served as a consultant for Servier. MB has received grant/ research support from the National Institutes of Health $(\mathrm{NIH})$, Cooperative Research Centre, Simons Autism Foundation, Cancer Council of Victoria, Stanley Medical Research Foundation, MBF, NHMRC, Beyond Blue, Rotary Health, the Geelong Medical Research Foundation, Bristol-Myers Squibb, Eli Lilly, GlaxoSmithKline, Meat and Livestock Board, Organon, Novartis, MaynePharma, Servier, and Woolworths; has served as a speaker for AstraZeneca, Bristol-Myers Squibb, Eli Lilly, GlaxoSmithKline, Janssen-Cilag, Lundbeck, Merck, Pfizer, SanofiSynthelabo, Servier, Solvay, and Wyeth; and has served as a consultant for AstraZeneca, Bristol-Myers Squibb, Eli Lilly, GlaxoSmithKline, Janssen-Cilag, Lundbeck Merck, and Servier. The other authors report no conflicts of interest.

\section{References}

1 Berk M, Conus P, Kapczinski F, Andreazza AC, Yücel M, Wood SJ, et al. From neuroprogression to neuroprotection: implications for clinical care. Med J Aust. 2010;193:S36-40.

2 Berk M, Kapczinski F, Andreazza AC, Dean OM, Giorlando F, Maes $M$, et al. Pathways underlying neuroprogression in bipolar disorder: focus on inflammation, oxidative stress and neurotrophic factors. Neurosci Biobehav Rev. 2011;35:804-17.

3 Grande I, Magalhães PV, Kunz M, Vieta E, Kapczinski F. Mediators of allostasis and systemic toxicity in bipolar disorder. Physiol Behav. 2012;106:46-50.

4 Kapczinski F, Dias VV, Kauer-Sant'Anna M, Brietzke E, Vázquez $\mathrm{GH}$, Vieta $\mathrm{E}$, et al. The potential use of biomarkers as an adjunctive tool for staging bipolar disorder. Prog Neuropsychopharmacol Biol Psychiatry. 2009;33:1366-71

5 Vieta E, Popovic D, Rosa AR, Solé B, Grande I, Frey BN, et al. The clinical implications of cognitive impairment and allostatic load in bipolar disorder. Eur Psychiatry. 2013;28:21-9.
6 Kapczinski F, Dias VV, Kauer-Sant'Anna M, Frey BN, Grassi-Oliveira $\mathrm{R}$, Colom $\mathrm{F}$, et al. Clinical implications of a staging model for bipolar disorders. Expert Rev Neurother. 2009;9:957-66.

7 Aydemir O, Deveci A, Icelli I. Reliability and validity of the Turkish version of the structured interview guide for Hamilton Depression Rating Scale seasonal affective disorder. Psychiatr Turkey. 2006;8:18-21.

8 Karadag F, Oral ET, Yalcin AF, Erten E. [Reliability and validity of Turkish translation of Young Mania Rating Scale]. Turk Psikiyatri Derg. 2002;13:107-14.

9 Yatham LN, Torres IJ, Malhi GS, Frangou S, Glahn DC, Bearden CE, et al. The International Society for Bipolar Disorders-Battery for Assessment of Neurocognition (ISBD-BANC). Bipolar Disord. 2010;12:351-63.

10 Aydemir O, Eren I, Savaş H, Kalkan Oguzhanoglu N, Kocal N, Devrimci Ozguven $\mathrm{H}$, et al. [Development of a questionnaire to assess inter-episode functioning in bipolar disorder: Bipolar Disorder Functioning Questionnaire]. Turk Psikiyatri Derg. 2007;18:344-52.

11 Akyol O, Herken H, Uz E, Fadillioglu E, Unal S, Sögüt S, et al. The indices of endogenous oxidative and antioxidative processes in plasma from schizophrenic patients. The possible role of oxidant/ antioxidant imbalance. Prog Neuropsychopharmacol Biol Psychiatry. 2002;26:995-1005.

12 Kuloglu M, Ustundag B, Atmaca M, Canatan H, Tezcan AE, Cinkilinc $\mathrm{N}$. Lipid peroxidation and antioxidant enzyme levels in patients with schizophrenia and bipolar disorder. Cell Biochem Funct. 2002; 20:171-5

13 Kaya E, Aydemir O, Selcuki D. Residual symptoms in bipolar disorder: the effect of the last episode after remission. Prog Neuropsychopharmacol Biol Psychiatry. 2007;31:1387-92.

14 Mann-Wrobel MC, Carreno JT, Dickinson D. Meta-analysis of neuropsychological functioning in euthymic bipolar disorder: an update and investigation of moderator variables. Bipolar Disord. 2011;13:334-42.

15 Rosa AR, Reinares M, Franco C, Comes M, Torrent C, SánchezMoreno J, et al. Clinical predictors of functional outcome of bipolar patients in remission. Bipolar Disord. 2009;11:401-9.

16 Ozer S, Ulusahin A, Batur S, Kabakci E, Saka MC. Outcome measures of interepisode bipolar patients in a Turkish sample. Soc Psychiatry Psychiatr Epidemiol. 2002;37:31-7.

17 Goldberg JF. Adverse cognitive effects of psychotropic medications. In: Goldberg JF, Burdick KE, editors. Cognitive dysfunction in bipolar disorder: a guide for clinicians. Arlington: American Psychiatric Publishing; 2008. p. 137-58.

18 Sanchez-Moreno J, Martinez-Aran A, Tabarés-Seisdedos R, Torrent C Vieta E, Ayuso-Mateos JL. Functioning and disability in bipolar disorder: an extensive review. Psychother Psychosom. 2009;78:285-97.

19 Depp CA, Davis CE, Mittal D, Patterson TL, Jeste DV. Health-related quality of life and functioning of middle-aged and elderly adults with bipolar disorder. J Clin Psychiatry. 2006;67:215-21.

20 Bora E, Vahip S, Akdeniz F, Gonul AS, Eryavuz A, Ogut M, et al. The effect of previous psychotic mood episodes on cognitive impairment in euthymic bipolar patients. Bipolar Disord. 2007;9:468-77.

21 Berk M, Conus P, Lucas N, Hallam K, Malhi GS, Dodd S, et al. Setting the stage: from prodrome to treatment resistance in bipolar disorder. Bipolar Disord. 2007;9:671-8.

22 Berk M, Hallam KT, McGorry PD. The potential utility of a staging model as a course specifier: a bipolar disorder perspective. J Affect Disord. 2007;100:279-81.

23 Andreazza AC, Cassini C, Rosa AR, Leite MC, de Almeida LM, Nardin P, et al. Serum S100B and antioxidant enzymes in bipolar patients. J Psychiatr Res. 2007;41:523-9. 